\title{
JOINT SCALING TESTS
}

\author{
J. G. GALE, KENNETH MATHER and J. L. JINKS \\ Department of Genetics, University of Birmingham, Birmingham B15 2TT
}

Received 27.vii.76

\section{SUMmary}

It is shown that a joint scaling test developed by Tan (1974) is closely related to the widely used standard Cavalli joint scaling test (described by Mather and Jinks, 1971) which was not referred to in Tan's paper. With the numbers of individuals per generation observed in practice, the two tests give essentially similar results. The Cavalli procedure also provides estimates of genetical parameters and is more readily extended to a wider range of situations.

\section{Introduction}

FOR many years, a joint scaling test presented by Cavalli (1952) has been standard for testing the adequacy of an additive-dominance model when the basic data consist of the means of two inbred lines $\left(\mathrm{P}_{1}\right.$ and $\left.\mathrm{P}_{2}\right)$ and of the generations derived from them (usually $F_{1}, F_{2}, B_{1}$ and $B_{2}$ ). More recently, Tan (1974) has obtained a joint scaling test which at first sight appears quite different from that of Cavalli. Unfortunately, Tan does not refer to Cavalli's work, so that the apparent proliferation of tests may have puzzled some readers. The aim of this note is to clarify the relationship between the tests.

\section{The gavalli test}

Cavalli's procedure is set out in full by Mather and Jinks (1971, pp. 73-76), so that a brief summary only will be given here. If an additivedominance model is adequate to explain the data, the expected generation means are all linear functions of three parameters, $m,[d]$ and $[h]$. Let the estimated variance of the $i$ th generation, as found from a sample of size $n_{i}$, be $s_{i}^{2}$. The parameters $[m],[d]$ and $[h]$ are then readily estimated from the generation means by weighted least squares, using $w_{i}=n_{i} / s_{i}^{2}$ as the weight of the $i$ th generation mean. If our estimates are then inserted in the formulae for expected generation means, we can compare observed means $\bar{y}_{i}$ with estimated expected means $\hat{\mathscr{T}}_{i}$. If the variances $\sigma_{i}^{2}$ of the different generations were known exactly, so that we could put $w_{i}=n_{i} / \sigma_{i}^{2}$, the quantity

$$
Q=\sum_{i} w_{i}\left(\bar{y}_{i}-\hat{Y}_{i}\right)^{2}
$$

would (assuming, as is near enough correct, that the $\bar{y}_{i}$ are normally distributed) be a $\chi^{2}$ (with d.f. = number of generations less 3 ) testing the goodness of fit of our additive-dominance model. Since, however, the $\sigma_{i}^{2}$ are unknown, it has been necessary to take $w_{i}=n_{i} / s_{i}^{2}$, as we shall do throughout the ensuing discussion. Thus $Q$ will equal $\chi^{2}$ only 
approximately, although as we shall see the approximation is quite adequate in practice.

The Cavalli procedure extends quite easily to more complicated situations. Thus the number of generations may exceed the six described above. Moreover, should the simple additive-dominance model fail, a test of whether a revised model incorporating particular forms of digenic epistasis is adequate or whether further refinements such as trigenic epistasis or linkage are necessary is readily obtained (see Jinks and Perkins, 1969; Mather and Jinks, 1971, pp. 96-104). Whatever the model, we estimate the unknown parameters by weighted least squares and substitute these estimates in the formula for $Q$ given above; $Q$ is then approximately a $\chi^{2}$, with d.f. = number of generations less number of parameters estimated, testing the adequacy of the particular model assumed.

To facilitate comparison with Tan's approach, we shall express this last point in matrix notation. Let $\mathbf{y}$ be an $m \times \mathbf{l}$ matrix of the $m$ observed generation means and $\boldsymbol{\beta}$ a $p \times 1$ matrix of the $p$ unknown parameters, these matrices being related as:

$$
E(\mathbf{y})=\mathbf{X} \boldsymbol{\beta}
$$

where $\mathbf{X}$ is the "model " matrix.

Further, let $\mathbf{W}=\operatorname{diag}\left(w_{1}, w_{2} \ldots, w_{m}\right)$. Then

$$
Q=\mathbf{y}^{\prime}\left[\mathbf{W}-\mathbf{W X}\left(\mathbf{X}^{\prime} \mathbf{W} \mathbf{X}\right)^{-1} \mathbf{X}^{\prime} \mathbf{W}\right] \mathbf{y}
$$

is approximately a $\chi^{2}$ with $(m-p)$ d.f.

Tan has recovered our quantity $Q$ by a different method. He considers mainly the case where there are six generations $\left(\mathrm{P}_{1}, \mathrm{P}_{2}, \mathrm{~F}_{1}, \mathrm{~F}_{2}, \mathrm{~B}_{1}, \mathrm{~B}_{2}\right)$ and three unknown parameters $m,[d],[h]$. He starts with the quantities

$$
\begin{aligned}
& A=2 \bar{B}_{1}-\bar{P}_{1}-\bar{F}_{1} \\
& B=2 \bar{B}_{2}-\bar{P}_{2}-\bar{F}_{1} \\
& C=4 \bar{F}_{2}-2 \bar{F}_{1}-\bar{P}_{1}-\bar{P}_{2}
\end{aligned}
$$

(Mather, 1949; Mather and Jinks, 1971, pp. 71-73) It has long been recognised that these scaling criteria are correlated and indeed the Cavalli test was developed to overcome this difficulty. Tan considers the joint (multivariate normal) distribution of $A, B, C$. Let

so that

$$
\mathbf{z}=\left(\begin{array}{l}
A \\
B \\
C
\end{array}\right) \text { and } \mathbf{A}=\left(\begin{array}{rrrrrr}
-1 & . & -1 & . & 2 & . \\
. & -1 & -1 & . & . & 2 \\
-1 & -1 & -2 & 4 & . & .
\end{array}\right)
$$

$$
\mathbf{z}=\mathbf{A y}
$$

Let $\mathbf{V}$ be the covariance matrix of $\mathbf{z}$. The quantity

$$
\mathbf{z}^{\prime} \mathbf{V}^{-1} \mathbf{z}
$$

appearing in the exponent of the joint distribution is then a $\chi^{2}$ for 3 d.f. Replacing $\mathbf{V}^{-1}$ by its estimate $\mathbf{S}^{-1}$ obtained from

$$
\mathbf{S}=\mathbf{A} \mathbf{W}^{-1} \mathbf{A}^{\prime}
$$


he obtains

$$
T^{2}=\mathbf{z}^{\prime} \mathbf{S}^{-1} \mathbf{z}
$$

as an approximate $\chi_{3}^{2}$.

The relationship between Tan's $T^{2}$ and Cavalli's $Q$ becomes apparent when we consider the necessary properties of the matrix $\mathbf{A}$ which in turn follow from the properties of $A, B$ and $C$. Firstly, the efficacy of $A, B, C$ as scaling criteria depends on the fact that their expectations do not involve $m$, $[d]$ or $[h]$. Secondly, although $A, B, C$ are correlated, they are " distinct" in that they are linearly independent, i.e. no one criterion can be obtained as a linear combination of the other two, as is immediately apparent since each criterion involves one generation peculiar to itself. It may be helpful to note that, in view of this linear independence, $A, B$ and $C$ may, in principle, be transformed into three scaling criteria which are uncorrelated. For example, if all six generation means happened to have equal variances, it is easily verified that

$$
A+B, A-B, 3 A+3 B-7 C
$$

are uncorrelated inter se.

Turning now to the general case of $m$ generations, $p$ parameters, we shall, in order to use Tan's method, need to find $(m-p)$ linearly independent linear functions of the generation means, the functions being chosen so that their expectations do not involve the unknown parameters. These functions are then precisely analogous to scaling criteria. Writing them as an $(m-p) \times 1$ matrix $\mathbf{z}$, we have for the general case

$$
\mathbf{z}=\mathbf{A y}
$$

where $\mathbf{A}$ (which is analogous to our previous $\mathbf{A}$ ) is a matrix of rank $(m-p)$ and $\mathbf{A X}$ must equal a zero matrix in order to ensure that

$$
E(\mathbf{z})=E(\mathbf{A y})=\mathbf{A X} \boldsymbol{\beta} \text { shall }=\mathbf{0}
$$

It follows (see Wedderburn, 1974) that

$$
T^{2}=\mathbf{z}^{\prime}\left(\mathbf{A} \mathbf{W}^{-1} \mathbf{A}^{\prime}\right)^{-1} \mathbf{z}=\mathbf{y}^{\prime}\left[\mathbf{A}^{\prime}\left(\mathbf{A} \mathbf{W}^{-1} \mathbf{A}^{\prime}\right)^{-1} \mathbf{A}\right] \mathbf{y}
$$

which is Tan's $T^{2}$ for the general case equals Cavalli's $Q$ for the general case. Thus so far the two methods yield the same result.

However, it is apparent that Tan's method is much less convenient than Cavalli's since the former requires that we determine the matrix $\mathbf{A}$. In other words, we have to find an appropriate set of scaling criteria or functions analogous to scaling criteria. For example, with the $[m],[d],[h]$ model but with more than six generations, we must find scaling criteria $D, E, \ldots$ in addition to $A, B, C$. With more elaborate models, the construction of "scaling" criteria would be a matter of some difficulty. Since this difficulty does not arise with Cavalli's procedure, which also has the advantage of providing estimates of parameters, the Cavalli procedure is clearly better.

\section{Small samples}

When observed generation means are based on small samples, the estimated variances of the generation means may differ considerably from $38 / 1-D$ 
the true variances. This being so, the approximation $Q$ (or $T^{2}$ ) $=\chi^{\mathbf{2}}$ will not be as good as in the case of large samples. The novelty of Tan's approach lies in the attempt to produce a closer approximation to the distribution of $T^{2}$ than is given by the $\chi^{2}$ distribution. Tan does this by converting $T^{2}$ into a variance ratio. For example, for thc six generation, three parameter case, he puts

$$
\frac{f_{T}-2}{3 f_{T}} T^{2}=F\left(3, f_{T}-2\right)
$$

In this formula

$$
f_{T}=T^{4} /\left\{\sum_{i=1}^{6} \frac{1}{f_{i}}\left(\frac{s_{i}^{2}}{n_{i}} \mathbf{z}^{\prime} \mathbf{S}^{-1} \mathbf{A}_{i} \mathbf{A}_{i}^{\prime} \mathbf{S}^{-1} \mathbf{z}\right)^{2}\right\}
$$

where $f_{i}=n_{i}-1$ and $\mathbf{A}_{i}$ is the $i$ th column of $\mathbf{A}$ for this particular case. $F$ follows the usual $F$ distribution to a good approximation.

Although in principle this is an improvement, our numerical results given below indicate that, with sample sizes used in practice, the two methods give very similar answers, so that the use of $F$ rather than $\chi^{2}$ is by no means essential; hence previous results obtained by Cavalli's approach are sccure. We have had some difficulty in locating data in which the $n_{i}$ are small, since in the interests of precision experiments are seldom carried out with generations of less than 30 individuals and only in special circumstances would small samples be used. We are indebted to our colleagues Dr Caligari and Dr Pooni for supplying the two examples of data on final height in Nicotiana rustica given in table 1 ; the values of $n_{i}$ used in the second set of data are the lowest that we have ever known to be uscd in practice.

TABle 1

Two sels of data on final height in Nicotiana rustica

\begin{tabular}{crrrrrr} 
Generation & \multicolumn{3}{c}{ Set 1} & \multicolumn{3}{c}{ Set 2} \\
& $n_{i}$ & $\bar{y}_{i}$ & $s_{i}^{2}$ & $n_{i}$ & $\bar{y}_{i}$ & $s_{i}^{2}$ \\
$\mathrm{P}_{1}$ & 10 & $13 \cdot 955$ & $1 \cdot 6686$ & 10 & $11 \cdot 877$ & $0 \cdot 2044$ \\
$\mathrm{P}_{2}$ & 10 & $7 \cdot 575$ & $0 \cdot 6596$ & 10 & $12 \cdot 910$ & $0 \cdot 9454$ \\
$\mathrm{~F}_{1}$ & 20 & $11 \cdot 570$ & $0 \cdot 5630$ & 20 & $13 \cdot 547$ & $0 \cdot 6403$ \\
$\mathrm{~F}_{2}$ & 40 & $11 \cdot 501$ & $2 \cdot 1184$ & 40 & $12 \cdot 217$ & $2 \cdot 0899$ \\
$\mathrm{~B}_{1}$ & 40 & $13 \cdot 603$ & $3 \cdot 0806$ & 20 & $11 \cdot 840$ & $2 \cdot 5109$ \\
$\mathrm{~B}_{2}$ & 40 & $9 \cdot 219$ & $1 \cdot 2957$ & 20 & $13 \cdot 015$ & $2 \cdot 1087$
\end{tabular}

Results, with $(C)$ for Cavalli's method and $(T)$ for Tan's were as follows: Fxample 1 (C) $\chi_{3}^{2}=9.4378, \mathrm{P}=2.40 \%(\mathrm{~T}) \mathrm{F}(3,67)=3.0547, \mathrm{P}=3.43 \%$ Example $2(\mathrm{C}) \chi_{3}^{2}=12.5731, \mathrm{P}=0.57 \%(\mathrm{~T}) \mathrm{F}(3,83)=4.0924, \mathrm{P}=0.92 \%$

Thus although the Cavalli procedure slightly exaggeratcs the significance of departures of observed from expected, in neither sample is it in any way misleading, despite the unusually small number of individuals in the families.

We can explore the situation further by taking the $\bar{y}_{i}$ in sets 1 and 2 but varying either the $n_{i}$ or the $s_{i}^{2}$. Since our actual values of $n_{i}$ are about minimal in practice, it is worthwhile to consider only values of $n_{i}$ larger than those actually used in sets 1 and 2. Inspection of Tan's formula for 
$f_{T}$ shows that $f_{T}$ will increase essentially as $n_{i}^{3}$ so that the discrepancy between the values of $\mathrm{P}$ obtained by the two methods fails off very rapidly as the $n_{i}$ are increased. The $s_{i}^{2}$, not being under the experimenter's control, require more consideration. We give in table 2 , values of $\mathrm{P}$ obtained by taking the data in table 1 and dividing all the $s_{i}^{2}$ first by $0 \cdot 1$ and then by $0 \cdot 2,0 \cdot 4,0 \cdot 8,1 \cdot 6$ and $3 \cdot 2$ in turn.

TABLE 2

Effect of dividing all generation variances by a constant

$\begin{array}{ccc}\text { Divisor } & \overbrace{(C) \mathrm{P}(\%)}^{2} \text { Set } 1 \\ & 81.49 & 82 \cdot 13 \\ 0 \cdot 1 & 59 \cdot 61 & 61 \cdot 03 \\ 0.2 & 28.68 & 30.86 \\ 0.4 & 5.63 & 7 \cdot 16 \\ 0.8 & 0.17 & 0.39 \\ 1.6 & 0.0001 & 0.0020 \\ 3.2 & \end{array}$

\begin{tabular}{cc}
\multicolumn{2}{c}{ Set 2} \\
$(C) \mathrm{P}(\%)$ & $(T) \mathrm{P}(\%)$ \\
73.93 & 74.68 \\
47.27 & 48.73 \\
16.97 & $18 \cdot 71$ \\
1.81 & $2 \cdot 51$ \\
0.016 & 0.050 \\
\hline
\end{tabular}

We see that only when $P$ is so small that there can be no doubt of the departure of observed from expected is there any marked discrepancy between results from the two approaches, and even then they agree in showing that the null hypothesis is unacceptable. Thus we conclude that Tam's improvement is almost certainly marginal and that Cavalli's procedure is unlikely to be misleading in practice, even with families much smaller than those customarily used; with the larger families normally used the two tests virtually coincide in the results they give, so that no revision of conclusions based on Cavalli's procedure in earlier work is necessary. At the same time, the estimates of the unknown parameters $m,[d]$ and $[h]$ that Cavalli's procedure provides and its ready extension both to greater numbers of generations and to the testing of a wider range of models give a great advantage.

\section{REFERENCES}

CAVALLI, L. L. 1952. An analysis of linkage in quantitative inheritance. In Quantitative Inheritance, ed. E. C. R. Reeve, and C. H. Waddington. H.M.S.O., London.

JINKS, J. L., AND PERKINS, J. M. 1969. The detection of linked epistatic genes for a metrical trait. Heredity, 24, 465-475.

mather, K. 1949. The genetical theory of continuous variation. Proc. 8th Int. Cong. Genetics. Hereditas, Suppl. Vol., 376-401.

mather, K., AND Jinks, J. L. 1971. Biometrical Genetics. Chapman and Hall, London.

TAN, W, Y. 1974. The approximate overall test for epistatic effects in biometrical genetics. Biometrics, 30, 697-703.

WEDDERBURN, R. W. M. 1974. Generalised linear models specified in terms of constraints. J.R.S.S., B, 36, 449-454. 